\title{
An efficient ANN Based approach for Latent Fingerprint Matching
}

\author{
Jugal Kishor Gupta \\ Dr. K N Modi Institute of Engineering \& Tech., \\ Modinagar, Ghaziabad (India)
}

\author{
Rajendra Kumar \\ Vidya College of Engineering, \\ Meerut (India)
}

\begin{abstract}
Fingerprint matching is the process used to determine whether two sets of fingerprint ridge detail come from the same finger. There exist multiple algorithms that do fingerprint matching in many different ways. Some methods involve matching minutiae points between the two images, while others look for similarities in the bigger structure of the fingerprint. A major approach for fingerprint recognition today is to extract minutiae from fingerprint images and to perform fingerprint matching based on the number of corresponding minutiae pairings. One of the most difficult problems in fingerprint recognition has been that the recognition performance is significantly influenced by fingertip surface condition, which may vary depending on environmental or personal causes. In this paper we propose a method for offline fingerprint matching based on minutiae matching. However, unlike conventional minutiae matching algorithms, our algorithm also takes into account region and line structures that exist between minutiae pairs. This allows filling the small breaks in the curves created because of uneven surface and uneven pressure.
\end{abstract}

Keywords : minutiae, ridge, m_curve, reference point.

\section{INTRODUCTION}

Biometric authentication [1] has been receiving extensive attention over the past decade with increasing demands in automated personal identification. Biometrics is to identify individuals using physiological or behavioral characteristics, such as fingerprint, face, iris, retina, palm-print, etc. Among all the biometric techniques, fingerprint recognition is the most popular method and is successfully used in many applications. Typical fingerprint recognition methods employ feature-based image matching, where minutiae (i.e., ridge ending and ridge bifurcation) are extracted from the registered fingerprint image and the input fingerprint image, and the number of corresponding minutiae pairings between the two images is used to recognize a valid fingerprint image. The featurebased matching provides an effective way of identification for majority of people.

However, it has been known that there are a number of people whose fingerprints could not be identified by the feature based methods due to special skin conditions, where feature points are hard to be extracted by image processing. The ratio of people who have such difficult fingerprints varies depending on race, sex, age, job groupings, etc., but it is said that one to five percentage of total population may fall into this category. Addressing this problem, this paper we consider minutiae based model for offline finger print matching.

The fingerprints obtained from the crime scenes are of very bad quality because these are left unintentionally. Such fingerprints are called latent fingerprints. This paper investigates the major challenges in latent fingerprint recognition obtained from a crime scene. Poor quality fingerprint images lead to missing and spurious minutiae that degrade the performance of fingerprint matching system. The importance of image processing concepts cannot be ruled out to make a biometric method robust. However, the performance of a minutiae extraction algorithm relies heavily on the quality of the input fingerprint images. Some special treatment is done to reveal such fingerprints. The photo graph taken from different distances, uneven surface, different finger pressure, dust particles pose problems during recognition process. Improper finger pressure and uneven surface are the major cause to produce breaks in curves in a fingerprint. In order to improve the performance of the system, many researchers have been made efforts on the image enhancement algorithms. Most of the fingerprint recognition algorithms are based on minutia matching features. Therefore, minutiae extraction is one of the important steps in fingerprint verification algorithms. In this paper we present an algorithm to fill the broken curves on a fingerprint due to low finger pressure and uneven surface. Our proposed approach eliminates false minutiae that connect broken curves in fingerprint.

\section{RELATED WORK}

G. Sambasiva Rao et al. [2], proposed fingerprint identification technique using a gray level watershed method to find out the ridges present on a fingerprint image by directly scanned fingerprints or inked impression. Robert Hastings [3] developed a method for enhancing the ridge pattern by using a process of oriented diffusion by adaptation of anisotropic diffusion to smooth the image in the direction parallel to the ridge flow. The image intensity varies smoothly as one traverse along the ridges or valleys by removing most of the small irregularities and breaks but with the identity of the individual ridges and valleys preserved. Jinwei $\mathrm{Gu}$, et al.[4], proposed a method for fingerprint verification which includes both minutiae and model based orientation field is used. It gives robust discriminatory information other than minutiae points. Fingerprint matching is done by combining the decisions of the matchers based on the orientation field and minutiae.

Eric P. Kukula, et al.[5], purposed a method to investigate the effect of five different force levels on fingerprint matching performance, image quality scores, and minutiae count between optical and capacitance fingerprint sensors. Three images were collected from the right index fingers of 75 participants for each sensing technology. Descriptive statistics, analysis of variance, and Kruskal-Wallis nonparametric tests were conducted to assess significant differences in minutiae counts and image quality scores based on the force level.

The results reveal a significant difference in image quality score based on the force level and each sensor technology, yet there is no significant difference in minutiae count based on the force levels of the capacitance sensor. The image quality score, shown to be effected by force and sensor type, is one of many factors that influence the system matching performance, yet the removal of low quality images does not improve the system performance at each force level.

Lie Wei [6] proposed a method for rapid singularities searching algorithm which uses delta field Poincare index and a rapid classification algorithm to classify the fingerprint in to 5 classes. The detection algorithm searches the direction field which has the larger direction changes to get the singularities. 
Prabhakar S, Jain. A.K. et al. [7], has developed filter-based representation technique for fingerprint identification. The technique exploits both local and global characteristics in a fingerprint to make identification. Each fingerprint image is filtered in a number of directions and a 640dimensinal feature vector is extracted in the central region of the fingerprint. The feature vector is compact and requires only 640 bytes. The matching stage computes the Euclidian distance [8] between the template finger code and the input finger code. The method gives good matching with high accuracy. Ballan $M$ [9] introduced Directional Fingerprint Processing using fingerprint smoothing, classification and identification based on the singular points (delta and core points) obtained from the directional histograms of a fingerprint. A work [10] has been done to fill the curve breaks in linear fashion.

\section{OUR MODEL AND APPROACH}

In this paper we propose a method for filling of broken curves in non linear fashion using artificial intelligence. Many known algorithms have been developed for minutiae extraction based on orientation and gradients of the orientation fields of the ridges. In this paper we adopt the method used by Leung [11] where minutiae are extracted using feed forward artificial neural networks. The building blocks of a fingerprint recognition system are:

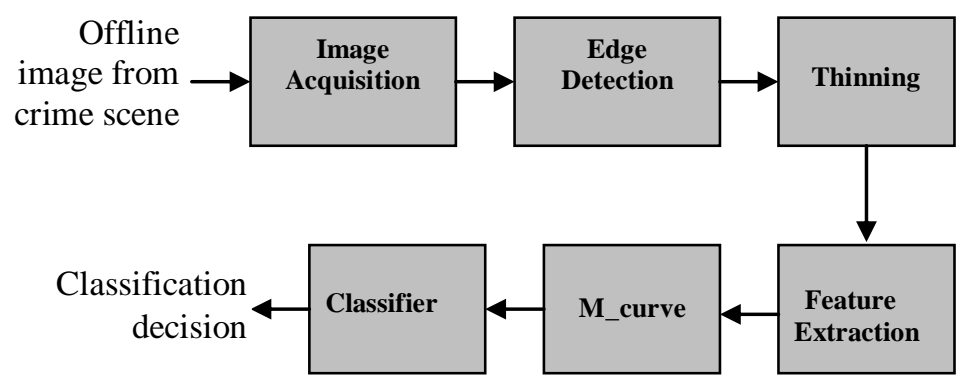

Fig. 1. Fingerprint recognition system

Image Acquisition: A number of methods are used to acquire fingerprints. Among them, the inked impression method remains the most popular one. Inkless fingerprint scanners are also present eliminating the intermediate digitization process. Fingerprint quality is very important since it affects directly the minutiae extraction algorithm. Two types of degradation usually affect fingerprint images:

1) the ridge lines are not strictly continuous since they sometimes include small breaks (gaps);

2) parallel ridge lines are not always well separated due to the presence of cluttering noise.

The resolution of the scanned fingerprints must be 500 dpi while the size is $300 \times 300$.

Edge Detection: An edge is the boundary between two regions with relatively distinct gray level properties. The idea underlying most edgedetection techniques is on the computation of a local derivative operator [12] such as 'Roberts', 'Prewitt' or 'Sobel' operators. In practice, the set of pixels obtained from the edge detection algorithm seldom characterizes a boundary completely because of noise, breaks in the boundary and other effects that introduce spurious intensity discontinuities. Thus, edge detection algorithms typically are followed by linking and other boundary detection procedures designed to assemble edge pixels into meaningful boundaries.

Thinning : An important approach to representing the structural shape of a plane region is to reduce it to a graph. This reduction may be accomplished by obtaining the skeleton of the region via thinning (also called skeletonizing) algorithm.
Feature Extraction: Extraction of appropriate features is one of the most important tasks for a recognition system. The feature extraction method [13] is explained below.

As shown in figure 2, the multilayer perceptron (MLP) of three layers is trained to detect the minutiae in the thinned fingerprint image of size 300x300. The first layer of the network has nine neurons associated with the components of the input vector. The hidden layer has five neurons and the output layer has one neuron. The network is trained to output a " 1 " when the input window in centered on a minutiae and a " 0 " when it is not. Figure 3 shows the initial training patterns which are composed of 16 samples of bifurcations in eight different orientations and 35 samples of nonbifurcations. The networking is trained using:

- The back propagation algorithm [14] with momentum and learning rate of 0.3 .

- $\quad$ The Al-Alaoui back propagation algorithm [15].

State the number of epochs needed for convergence as well as the training time for the two methods. Once the network is trained, the next step is to input the prototype fingerprint images to extract the minutiae.

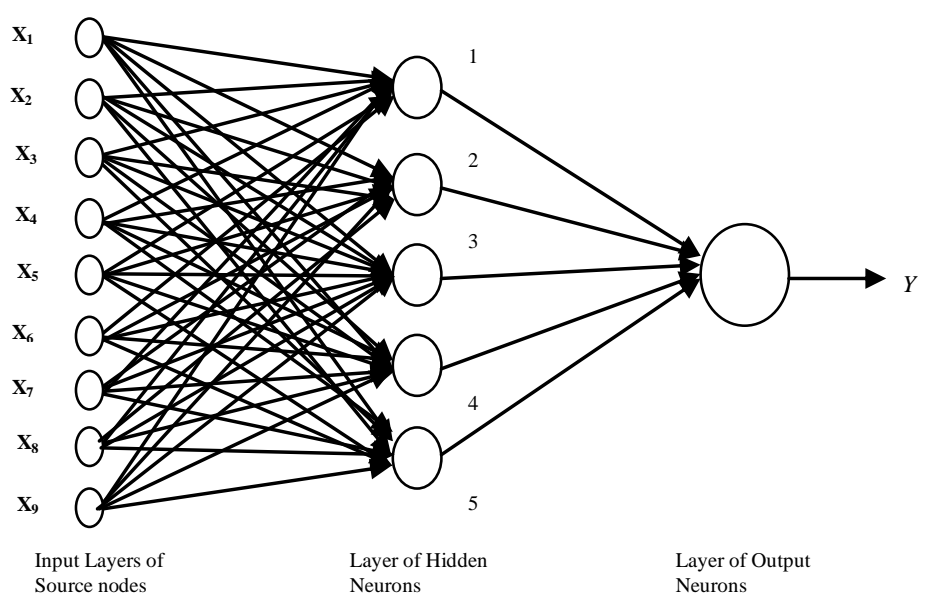

Fig. 2 Multilayer Perceptron Model

Detection of Bifurcations and Non-Bifurcations - The center of the fingerprint image was used to calculate the Euclidean distance between the center and the feature point. Usually, the center or reference point of the fingerprint image is what is called the "core" point. A core point, is located at the approximate center, is defined as the topmost point on the innermost upwardly curving ridgeline. The human fingerprint is comprised of various types of ridge patterns, traditionally classified according to the decades-old Henry system: left loop, right loop, arch, whorl, and tented arch. Loops make up nearly $2 / 3$ of all fingerprints, whorls are nearly $1 / 3$, and perhaps $5-10 \%$ are arches. Figure 4 shows some fingerprint patterns with the core point are marked. Many singularity points detection algorithms were investigated to locate core points, among them the famous "Poincaré" index method $[4,5]$ and the one described in [6]. For simplicity we will assume that the core point is located at the center of the fingerprint image.

After extracting the location of the minutiae for the prototype fingerprint images, the calculated distances will be stored in the database along with the ID or name of the person to whom each 
fingerprint belongs. The last phase is the verification phase where testing fingerprint image:

1. is inputted to the system

2. minutiae are extracted

3. Minutiae matching: comparing the distances extracted minutiae to the one stored in the database

4. Identify the person

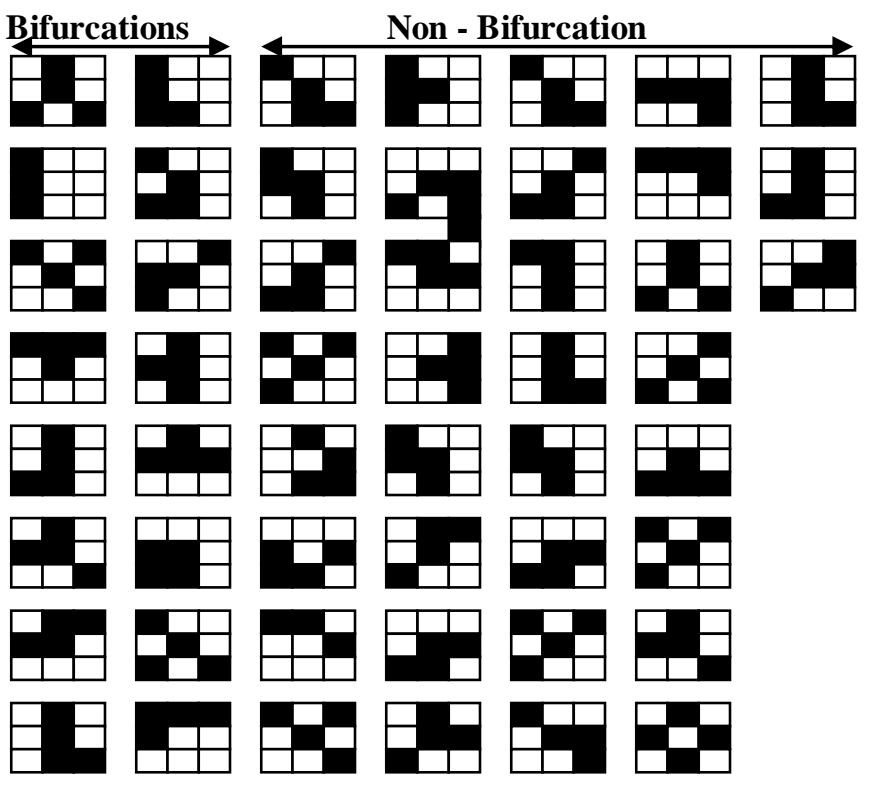

Fig. 3. Training set

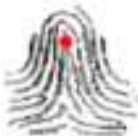

(a)

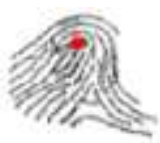

(b)

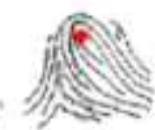

(c)

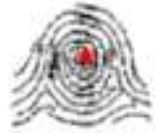

(d)
Fig. 4. Core points on different fingerprint patterns. (a) tented arch, (b) right loop, (c) left loop, (d) whorl

$\boldsymbol{m}$-curve - The basic reason behind false minutiae is the presence of dust particles, oily fingers, dry fingers, and cut on finger. Dry finger may fail to produce a complete line/curve up to the actual end points. As a result the break points create false (invalid) minutiae. These false minutiae significantly affect the accuracy of matching if they are simply regarded as genuine minutia. Therefore, some algorithms of removing false minutia are crucial to keep the fingerprint verification system efficient. Following 16 equations are applied for increasing the curves length -

if $P(i, j)=1 \& P(i+1, j-1)=1 \& P(i, j-2)=1$ then $P(i-1, j-3)=1$

if $P(i, j)=1 \& P(i+1, j+1)=1 \& P(i, j+2)=1$ then $P(i-1, j+3)=1 \quad \ldots(2)$

if $P(i, j)=1 \& P(i-1, j-1)=1 \& P(i, j-2)=1$ then $P(i+1, j-3)=1 \quad \ldots$ (3)

if $P(i, j)=1 \& P(i-1, j+1)=1 \& P(i, j+2)=1$ then $P(i+1, j+3)=1 \quad \ldots(4)$

if $P(i, j)=1 \& P(i+1, j-1)=1 \& P(i+2, j)=1$ then $P(i+3, j+1)=1 \quad \ldots(5)$

if $P(i, j)=1 \& P(i-1, j-1)=1 \& P(i-2, j)=1$ then $P(i-3, j+1)=1 \quad \ldots(6)$

if $P(i, j)=1 \& P(i+1, j+1)=1 \& P(i+2, j)=1$ then $P(i+3, j-1)=1 \quad \ldots(7)$

if $P(i, j)=1 \& P(i-1, j-1)=1 \& P(i-2, j)=1$ then $P(i-3, j+1)=1 \quad \ldots(8)$

if $P(i, j)=1 \& P(i, j-1)=1 \& P(i+1, j-2)=1$ then $P(i+2, j-2)=1 \quad \ldots(9)$

if $P(i, j)=1 \& P(i-1, j+1)=1 \& P(i-1, j+2)=1$ then $P(i, j+3)=1 \quad \ldots(10)$

if $P(i, j)=1 \& P(i+1, j)=1 \& P(i+2, j-1)=1$ then $P(i+2, j-2)=1 \quad \ldots(11)$

if $P(i, j)=1 \& P(i, j+1)=1 \& P(i-1, j+2)=1$ then $P(i-2, j+2)=1 \quad \ldots(12)$

if $P(i, j)=1 \& P(i+1, j+1)=1 \& P(i+2, j+2)=1 \& P(i+2, j+3)=1$

then $P(i+2, j+4)=1$

if $P(i, j)=1 \& P(i-1, j+1)=1 \& P(i-2, j-2)=1 \& P(i-3, j-2)=1$

then $P(i-4, \mathrm{j}-2)=1$

if $P(i, j)=1 \& P(i-1, j-1)=1 \& P(i-2, j-2)=1 \& P(i-1, j-3)=1$ then $P(i, j-4)=1$

if $P(i, j)=1 \& P(i+1, j+1)=1 \& P(i+2, j+2)=1 \& P(i+3, j+2)=1$

then $P(i+4, j+2)=1$

The graphical presentation of above 16 equations is given by figure 5 .

\begin{tabular}{|l|l|l|l|}
\hline 1 & 2 & 3 & 4 \\
\hline 5 & 6 & 7 & 8 \\
\hline 9 & 10 & 11 & 12 \\
\hline 13 & 14 & 15 & 16 \\
\hline
\end{tabular}

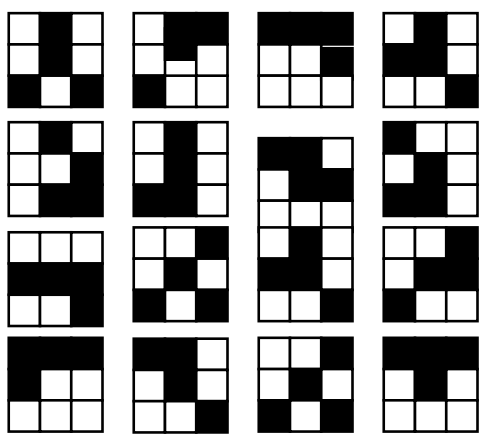

Fig. 5. Illustration of equations 1 through 16

Classifier: After scanning the entire fingerprint image, the resulting output is a binary image revealing the location of minutiae. In order to prevent any falsely reported output and select "significant" minutiae, two more rules are added to enhance the robustness of the algorithm:

1) At those potential minutiae detected points, we reexamine them by increasing the window size by $5 \times 5$ and scanning the output image.

2) If two or more minutiae are to close together (few pixels away) we ignore all of them.

To insure translation, rotation and scale-invariance, the following operations will be performed:

The Euclidean distance $d(i)$ from each minutiae detected point to the center is calculated. The referencing of the distance data to the center point guarantees the property of positional invariance. The data will be sorted in ascending order from $d(0)$ to $d(N)$, where $N$ is the number of detected minutiae points, assuring rotational invariance. The data is then normalized to unity by shortest distance $d(0)$, i.e., $\operatorname{dnorm}(i)=d(0) / d(i)$; This assures scale invariance property.

\section{IMPLEMENTATION AND RESULTS}

For experimental we used database of 30 fingerprints of same finger at different pressure. We conducted total 3600 experiments. In 2700 out of 3600 sub experiments, favorable results were 1449, which is $53.67 \%$ shown by figure 6 . In total we have conducted 3600 sub experiments, 900 without $m$ _curve and 2700 by applying $m_{-}$curve. This way in $53.67 \%$ experiments, matching was improved or not altered.

Table 1 shows the summary of our experiments. It represents consolidated favorable results. In our experiments favorable results are sum of results producing better matching, no change in matching and the matching results decreasing within $10 \%$.

At low pressure we got fingerprints with false minutiae due to discontinuation of curves. To eliminate this kind of false minutiae we manipulated the finger print images by increasing the length of each curve having at least three pixels at terminations. This way the rate of successful matching is increased by $10 \%$ we performed. 


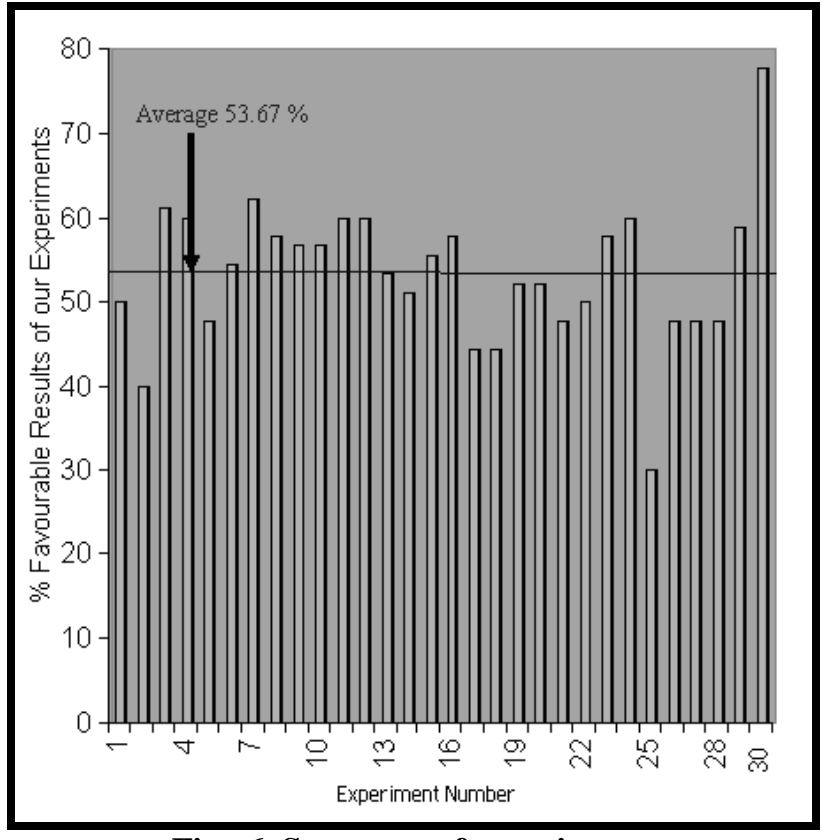

Fig. 6. Summary of experiments

Table 1, shows the summary of our experiments. It represents consolidated favorable results. In our experiments favorable results are sum of results producing better matching, no change in matching and the matching results decreasing within $10 \%$.

Table 1. Summary of experiments

\begin{tabular}{|c|c|c|c|c|c|c|}
\hline 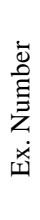 & 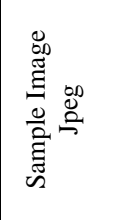 & 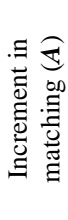 & 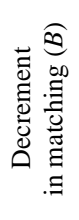 & 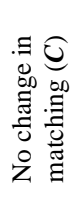 & 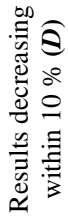 & 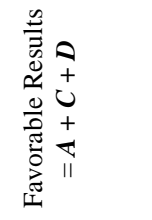 \\
\hline 01 & \begin{tabular}{|l|} 
p000_0.3 \\
\end{tabular} & 24 & 60 & 12 & 09 & $45(50.00 \%)$ \\
\hline 02 & \begin{tabular}{|l|} 
p004_0.4 \\
\end{tabular} & 31 & 55 & 04 & 01 & $36(40.00 \%)$ \\
\hline 03 & p008_0.5 & 43 & 42 & 05 & 07 & $55(61.11 \%)$ \\
\hline 04 & p012_0.6 & 38 & 42 & 10 & 06 & $54(60.00 \%)$ \\
\hline 05 & p016_0.7 & 31 & 56 & 03 & 09 & $43(47.77 \%)$ \\
\hline 06 & p018_0.8 & 35 & 49 & 06 & 08 & $49(54.44 \%)$ \\
\hline 07 & p020_0.9 & 49 & 35 & 06 & 01 & $56(62.22 \%)$ \\
\hline 08 & p028_1.0 & 40 & 38 & 12 & 07 & $52(57.77 \%)$ \\
\hline 09 & \begin{tabular}{|l|} 
p030_1.1 \\
\end{tabular} & 42 & 39 & 09 & 00 & $51(56.66 \%)$ \\
\hline 10 & p036_1.2 & 33 & 39 & 18 & 07 & $51(56.66 \%)$ \\
\hline 11 & p042_1.3 & 41 & 40 & 09 & 04 & $54(60.00 \%)$ \\
\hline 12 & $\begin{array}{l}\text { p052_1.4 } \\
\end{array}$ & 45 & 41 & 04 & 05 & $54(60.00 \%)$ \\
\hline 13 & p055_1.5 & 39 & 48 & 03 & 06 & $48(53.33 \%)$ \\
\hline 14 & p058_1.6 & 34 & 53 & 03 & 09 & $46(51.11 \%)$ \\
\hline 15 & p060_1.7 & 41 & 46 & 03 & 06 & $50(55.55 \%)$ \\
\hline 16 & p062_1.8 & 39 & 42 & 09 & 04 & $52(57.77 \%)$ \\
\hline 17 & p064_1.9 & 30 & 55 & 05 & 05 & $46(51.11 \%)$ \\
\hline 18 & p066_2.0 & 26 & 53 & 11 & 03 & $40(44.44 \%)$ \\
\hline 19 & p068_2.1 & 35 & 46 & 09 & 03 & $47(52.22 \%)$ \\
\hline 20 & p070_2.2 & 42 & 45 & 03 & 02 & $47(52.22 \%)$ \\
\hline 21 & p074_2.3 & 36 & 50 & 04 & 03 & $43(47.77 \%)$ \\
\hline 22 & p076_2.4 & 32 & 48 & 10 & 03 & $45(50.00 \%)$ \\
\hline 23 & p082_2.5 & 38 & 49 & 03 & 11 & $52(57.77 \%)$ \\
\hline 24 & p083_2.6 & 24 & 36 & 30 & 00 & $54(60.00 \%)$ \\
\hline 25 & p092_2.7 & 14 & 60 & 06 & 07 & $27(30.00 \%)$ \\
\hline 26 & p094_2.8 & 35 & 49 & 06 & 02 & $43(47.77 \%)$ \\
\hline 27 & \begin{tabular}{|l|l} 
p100_2.9 \\
\end{tabular} & 38 & 49 & 03 & 02 & $43(47.77 \%)$ \\
\hline 28 & p104_3.0 & 36 & 47 & 07 & 00 & $43(47.77 \%)$ \\
\hline 29 & p106_3.1 & 45 & 42 & 03 & 05 & $53(58.88 \%)$ \\
\hline
\end{tabular}

\begin{tabular}{|l|l|l|l|l|l|l|}
\hline 30 & $\mathrm{p} 110.3 .2$ & 40 & 36 & 14 & 16 & $70(77.77 \%)$ \\
\hline
\end{tabular}

\section{CONCLUSION}

The performance of fingerprint identification system relies critically on the image quality. Hence, good quality images make the system performance more robust. However, it is very difficult to obtain good quality images practically. To overcome this problem, image enhancement/manipulation steps are applied. We used artificial neural network for filling the gaps in fingerprints appeared due to low pressure and uneven surface. Our matching results were increased in $53.67 \%$ cases. During the experiments we have seen that if the finger pressure difference between the images of the same finger was $\pm 10 \%$ we got $98 \%$ result for true matching.

\section{REFERENCES}

[1] Alan E. Zuckerman, M.D., Kenneth A. Moon, M.D., Kenneth Eaddy, "Comparison of Fingerprint and Iris Biometric Authentication for Control of Digital Signatures", Annual Symposium Proceedings, AMIA 2002.

[2] G. Sambasiva Rao et al., "Fingerprint Recognition using Minutia Score Matching", International Journal of Engineering Science and Technology Vol. (2), p.p. 35-42, 2009.

[3] Robert Hastings, "Ridge Enhancement in Fingerprint Images Using Oriented Diffusion”, 9th Biennial Conference of the Australian Pattern Recognition Society on Digital Image Computing Techniques and Applications (DICTA 2007).

[4] Ravi. J. et al , "Fingerprint Recognition using Minutia Score Matching", International Journal of Engineering Science and Technology Vol. 1(2), pp. 35-42, 2009.

[5] Eric P. Kukula, "Impact of Gender on Fingerprint Recognition Systems", $5^{\text {th }}$ International Conference on Information Technology and Applications (ICITA 2008).

[6] Li Wei, "Constrained nonlinear models of fingerprint orientations with prediction", ACM Journal of Pattern Recognition, Volume 39, Issue 1, 2006.

[7] www.ijest.info/docs/IJEST09-01-02-02.pdf

[8] Salil Prabhakar, Anil Jain. "Fingerprint Recognition by Euclidean Distance", Second International Conference on Computer and Network Technology, 2010.

[9] Ballan M, "A Fingerprint Classification Technique Using Directional Images Signal", International Conference on System and Computer, Berkley, California, pp. 95 - 99, 2003.

[10] Rajendra Kumar, "Variation in Biometric Data: Modeling and Simulation", M.Tech. Thesis, UPTU 2007.

[11] F.A. Afsar, M. Arif and M. Hussain, "Fingerprint Identification and Verification System using Minutiae Matching", National Conference on Emerging Technologies, 2004.

[12] N Suriyanarayanan, "Performance measure of Local derivative operator in Fingerprint Detection", Academic Open Internet Journal, ISSN 1311-4360 Vol. 23, 2008.

[13] V Humbe, "Mathematical Morphology Approach for Genuine Fingerprint feature extraction method", International Conference on Pattern Recognition, 2008.

[14] V. V. Phansalkar, and P. S. Sastrq, "Analysis of the BackPropagation Algorithm with Momentum", IEEE Transactions on Neural Networks, Vol. 5. No. 3, 1994.

15] Suzan A. Mahmood, "Identification Using Backpropagation Neural Network", Journal of Zankoy Sulaimani, 2008. 